\title{
New ideas in the theory of core-collapse supernova explosions
}

\author{
Adam Burrows ${ }^{* \dagger}$ \\ Department of Astronomy, University of Arizona \\ E-mail: burrowseas.arizona.edu

\section{Luc Dessart} \\ Department of Astronomy, University of Arizona \\ E-mail: Luceas.arizona.edu
}

\section{Eli Livne}

Racah Institute of Physics, The Hebrew University

E-mail: eli@frodo.fiz.huji.ac.il

\section{Christian D. Ott}

Max-Planck-Institut für Gravitationsphysik, Albert-Einstein-Institut

E-mail: cott@aei.mpg.de

\begin{abstract}
We summarize some of the new results emerging from our recent multi-dimensional simulations of core collapse and explosion. We see a new role for rapid core oscillations and acoustic power in igniting asymmetrical supernova explosions and obtain explosions for all progenitors studied. Furthermore, we have explored the effects of rapid rotation and have estimated the degree of anisotropy of the emergent neutrino emissions in the generic context. All these aspects bear on the mechanisms of explosion and pulsar proper motion. While more detailed calculations are called for, if any of the newly-identified phenomena survive, we suggest that the field of supernova theory may be entering a new and productive phase.
\end{abstract}

International Symposium on Nuclear Astrophysics - Nuclei in the Cosmos - IX

25-30 June 2006

CERN

\footnotetext{
* Speaker.

$\dagger$ This work supported in part by the US DOE under the SciDAC program, the US NSF, and the Joint Institute for Nuclear Astrophysics (JINA).
} 


\section{Possible Surprises in Supernova Theory}

The mechanism for the explosion of core-collapse supernovae has vexed theorists for decades. Though no definitive answers have achieved consensus, some new ideas have emerged from the recent generation of multi-dimensional computer simulations that have stimulated thought, and some controversy. None more so than the model proferred by Burrows et al.[1]. This model emerges naturally from our 2D radiation hydrodynamic runs using the multi-group flux-limited diffusion (MGFLD) variant of VULCAN/2D and does not hinge upon neutrino heating to ignite explosion. Rather, acoustic radiation from the damping of core g-mode oscillations substitutes as the driver of explosion. First, an advective-acoustic oscillation à la Foglizzo[2, 3] with a period of 10-30 milliseconds (ms) begins in earnest hundreds of milliseconds after bounce. Its growth saturates due to the generation of secondary shocks, and kinks in the resulting shock structure funnel and regulate subsequent accretion onto the inner core. However, this instability is not the primary agent of explosion. Rather, it is the acoustic power generated early on in the inner turbulent region stirred by the accretion plumes, and most importantly, but later on, by the excitation and sonic damping of core g-mode oscillations. An $\ell=1$ mode with a period of $\sim 3$ ms grows at late times to be prominent around $\sim 500 \mathrm{~ms}$ after bounce. The accreting protoneutron star is a self-excited oscillator, tuned to the most easily excited core g-mode. The angular distribution of the emitted sound is fundamentally aspherical. The sound pulses radiated from the core steepen into shock waves that merge as they propagate into the outer mantle and deposit their energy and momentum with high efficiency. The ultimate source of the acoustic power is the gravitational energy of infall and the core oscillation acts like a transducer to convert this accretion energy into sound. An advantage of the acoustic mechanism is that acoustic power does not abate until accretion subsides, so that it is available as long as it may be needed to explode the star.

Whether all, some, or none of this phenomenology survives remains to be seen. Currently, there is no other supernova code that combines the features necessary to simulate this model: 1) multi-group, 2) general grid that can liberate the inner core to execute $\ell=1$ g-mode oscillations, 3) general gravity, and, we believe, 4) moving grid capability. Due to the finite-difference character of $2 \mathrm{D}$ codes that employ spherical coordinates all the way to the center, to the singularity in those coordinates at that center, and to the reflecting boundary condition frequently imposed at this center, spherical-coordinate codes are likely to inhibit core translational motions artificially and, hence, to inhibit the $\ell=1 \mathrm{~g}$-modes that are central to the mechanism we have identified. In fact, most multiD supernova codes either do the inner core in 1D, or excise the inner core altogether. Hence, testing the acoustic model will be a challenge.

Nevertheless, we present here in graphical form a subset (by no means exhaustive) of the special features that mark our recent multi-D simulations. First, in the left panel of Fig. 11 we present an equatorial slice of the mass density at early and late times for one of our collapse runs of the 15-M $\odot$ progenitor model of Woosley, Heger, \& Weaver[ $\llbracket$ (WHW02). The plus signs mark the zone positions. This figure serves to demonstrate that we maintain approximately five zones per decade in density even around the neutrinospheres near 30 kilometers $(\mathrm{km})$. Though better resolution is desirable, this resolution is not bad. On the right panel of Fig. 1 we depict the position(s) of the shock along the two poles of our axially-symmetric calculations for simulations of the 11.2, 13, and $20 \mathrm{M}_{\odot}$ models of WHW02. This slice provides a glimpse of the advective-acoustic 
oscillation of the shock (termed the Standing-Accretion-Shock-Instability, "SASI," by Blondin, Mezzacappa, \& DeMarino[[]]) before explosion. The SASI excursions $\geq 200$ milliseconds after bounce are large, the top and bottom hemispheres are not in phase, the dominant frequency is an increasing function of progenitor mass (actually, accretion rate while stalled[[]) and ranges from $\sim 30$ to $\sim 80 \mathrm{Hertz}$, and the average radius of the shock is larger for the smallest progenitor. The vigorous stirring and turbulence generated by the SASI sets the stage for the excitation of the core oscillations and explosion by the core acoustic model. The left-hand-side and right-hand-side of Fig. Z portray maps of the entropy distributions along the poles (plus $\mathrm{z}$ and minus $\mathrm{z}$ in our cylindrical coordinate system) versus time for a simulation of the 11.2 and the $20 \mathrm{M}_{\odot}$ models of WHW02. These plots cover the SASI phase to the onset and early development of the explosion. High entropies (dark) generated by the superposition of multiple shocks and neutrino heating are an index of explosion. Note that the delay to explosion is not short and that the two models explode in opposite directions (though the $11.2-\mathrm{M}_{\odot}$ model is more symmetric). The direction of explosion is stochastic and completely determined by chaos and chance.

As an aside, we show in the left panel of Fig. 3 the magnetic fields that would be necessary to compete with the local pressure in the polar directions. The darkest regions (highest fields) obtain in the center, where the pressure is very high. Note that fields as high as $10^{14}$ gauss would be called for even at radii as large as $200 \mathrm{~km}$ and that fields as high as $10^{17-18}$ gauss would be required in the centers. Such high fields $(\beta=1)$ might obtain for the most rapidly rotating progenitors[6], particularly at radii of 30-300 km, but for slowly rotating progenitors, which seem generic [7], they are unlikely to be generated.

On the right-hand-side of Fig. 3, we provide a 3D rendition at a time just after bounce, but before explosion, of the simulation by Dessart et al. [8] of the dynamics of a white dwarf in the context of accretion-induced-collapse (AIC). Such models achieve the Chandrasekhar mass and rapid rotation by accretion from a stellar companion. The surfaces are isodensity contours and the arrows are velocity vectors, with rotation included. The collapse proceeds mostly along the poles where the centrifugal effect is minimal. The equatorial material is soon supported in quasi-Keplerian orbits in the inner regions and constitutes the early disk. Within 100 milliseconds of bounce, the explosion (not shown) blasts asymmetrically along the poles; within $\sim 250-300$ milliseconds, wide-angle, bipolar, neutrino-driven jets emerge. Due to rapid rotation of the inner core, the neutrinospheres are grossly aspherical and the core is quite oblate (with 1:2 - 1:3 axis ratios). As Fig. 3 indicates, VULCAN/2D is capable of incorporating modest or extreme rotational effects, depending on the initial rotational structure of the progenitor. This is a useful feature.

Finally, we show on the left in Fig. 4 the force on the inner core due to neutrino recoil and on the right the corresponding impulse (time integral of the force) versus time during the first postbounce second of our simulation of the 11-M $\odot$ model of Woosley \& Weaver[ 9 . The MGFLD version of VULCAN/2D is uniquely capable of providing semi-realistic value for these quantities. We find that during the pre-explosion phase, the neutrino recoil averages to small values, but that with explosion the neutrinos emerge anisotropically. In our axisymmetric calculations, the neutrino flux for all species is higher and hotter in the direction of explosion. As a result, the recoil effects of both neutrinos and matter $a d d$. This phenomenology provides a natural mechanism for pulsar kicks: the accumulating neutrino impulse and the asymmetric matter blast impart a systematic recoil to the residual protoneutron star, in a fashion that is quite natural for a top-bottom asymmetric explosion. 
This recoil grows over a long time (many seconds, as inferred from our simulations). Which effect dominates, the matter recoil or the neutrino recoil, remains to be determined, but at this stage in our theoretical explorations either could.

What the actual contributions of sound and neutrinos are to the supernova phenomenon as a function of progenitor remains to be determined and will require even more sophisticated numerical tools than we have applied to date to reach an accepted answer. However, we see in our current suite of multi-D calculations a possibly pivotal role for mechanical and acoustic power and for core motions.

\section{References}

[1] A. Burrows, E. Livne, L. Dessart, C.D. Ott, \& J. Murphy, A New Mechanism for Core-Collapse Supernova Explosions, Ap. J. 640 (878) 2006 [astro-ph/ 0510687 ].

[2] T. Foglizzo, P., Galletti, P., L. Scheck, \& H.-T. Janka, Instability of a stalled accretion shock: evidence for the advective-acoustic cycle, submitted to Ap. J. 2006, astro-ph/0606640.

[3] J.M. Blondin, A. Mezzacappa, \& C. DeMarino, Stability of Standing Accretion Shocks, With an Eye Toward Core Collapse Supernovae, Ap. J. 584 (971) 2003 [ast ro-ph / 0210634 ].

[4] S.E. Woosley, A. Heger, \& T.A. Weaver, The evolution and explosion of massive stars, Rev. Mod. Phys. 74 (1015) 2002.

[5] A. Burrows, E. Livne, L. Dessart, C.D. Ott, \& J. Murphy, Features of the Acoustic Mechanism of Core-Collapse Supernova Explosions, submitted to Ap. J. , $2006 \mathrm{~b}$.

[6] S. Akiyama, J.C. Wheeler, D.L. Meier, \& I. Lichtenstadt, The Magnetorotational Instability in Core-Collapse Supernova Explosions, Ap. J. 584 (954) 2003 [astro-ph/0208128].

[7] C.D. Ott, A. Burrows, L. Dessart, \& E. Livne, The Spin Periods and Rotational Profiles of Neutron Stars at Birth, Ap. J. 164 (130) 2006 [astro-ph/ 0508462 ].

[8] L. Dessart, A. Burrows, C.D. Ott, C.D., E. Livne, S.-Y. Yoon, \& N. Langer, Multi-Dimensional Simulations of the Accretion-Induced Collapse of White Dwarfs to Neutron Stars, Ap. J. 644 (1063) 2006 [astro-ph/0601603].

[9] S.E. Woosley \& T.A. Weaver, The Evolution and Explosion of Massive Stars. II. Explosive Hydrodynamics and Nucleosynthesis, Ap. J. Suppl. 101 (181) 1995. 

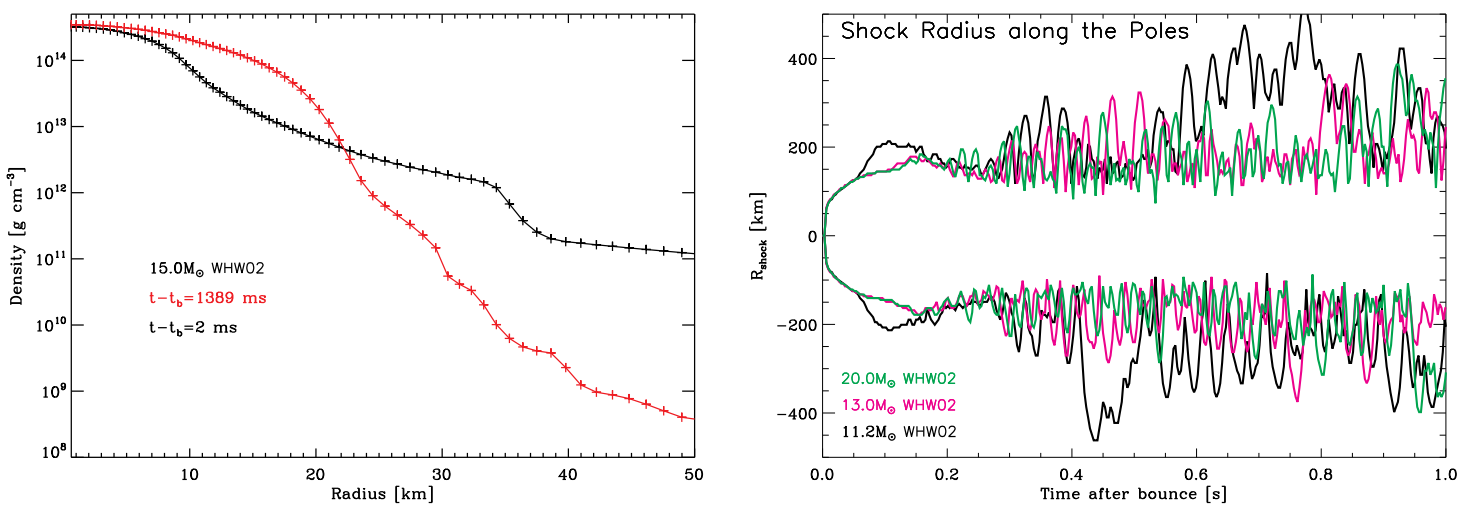

Figure 1: Left: Illustration of the temporal evolution of the equatorial density profiles at selected postbounce times (earlier is darker), for the 15-M $\odot$ model of WHW02. Symbols correspond to the VULCAN/2D grid-point locations along the equatorial direction and in this specific run. Although the resolution could be improved, our standard grid setup allows the coverage of every density decade with at least 5 points, even at late times. Right: Time evolution of the shock radius along the poles for our VULCAN/2D simulations of

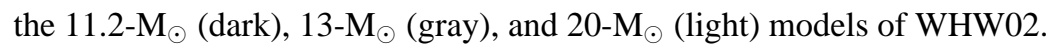
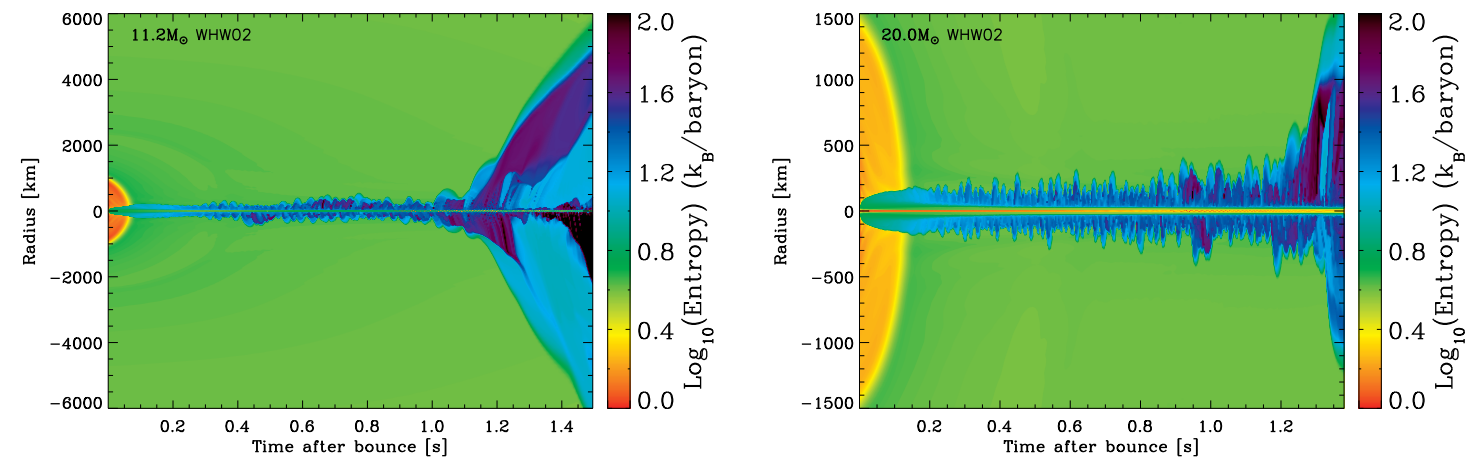

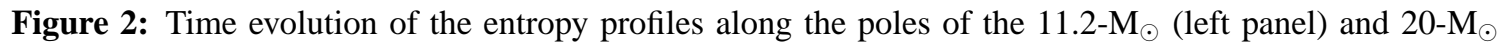
(right panel) models of WHW02, as calculated by Burrows et al.[1], 5]. The positions of the shocks are clearly indicated by the abrupt transition from the low entropy (light shaded region) of the infalling material. Grayscale bars indicating the values of the logarithm of the entropy (per baryon per Boltzmann constant) are provided on the right-hand-sides of each panel and go from light (entropy $\sim 1$ ) to dark (entropy $\geq 100$ per baryon per Boltzmann's constant). 

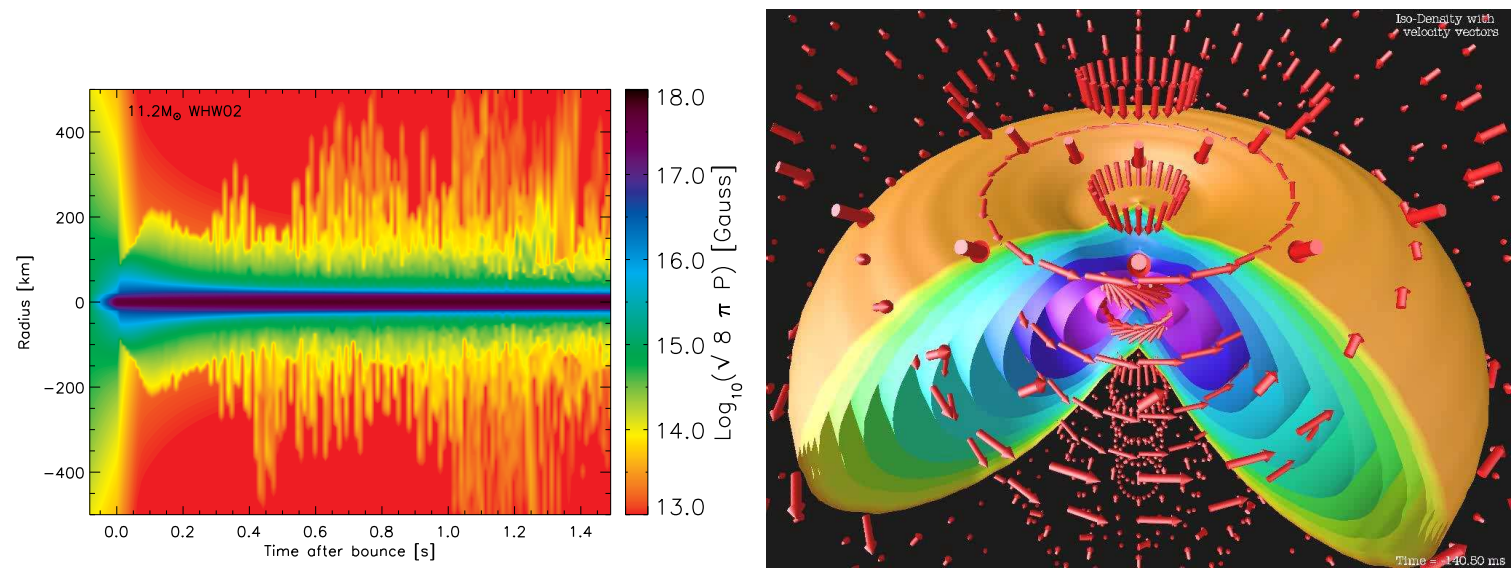

Figure 3: Left: Grayscale of the time evolution after bounce of the quantity $\sqrt{(} 8 \pi P)$ (in gauss), where $P$ is the thermal pressure, for the $11.2-\mathrm{M}_{\odot}$ model of WHW02. This quantity corresponds to the value of the local magnetic field required to have equal thermal and magnetic pressure contributions (a plasma $\beta$ of unity). Right: A snapshot employing isodensity contours during the immediate post-bounce phase of the simulation of a rapidly rotating white dwarf created in accretion-induced collapse. The arrows indicate the direction and magnitude of the velocity vectors, including the effect of rotation. This model soon explodes along the poles, leaving behind a thick disk of material (Simulation performed and published by Dessart et al.[8].
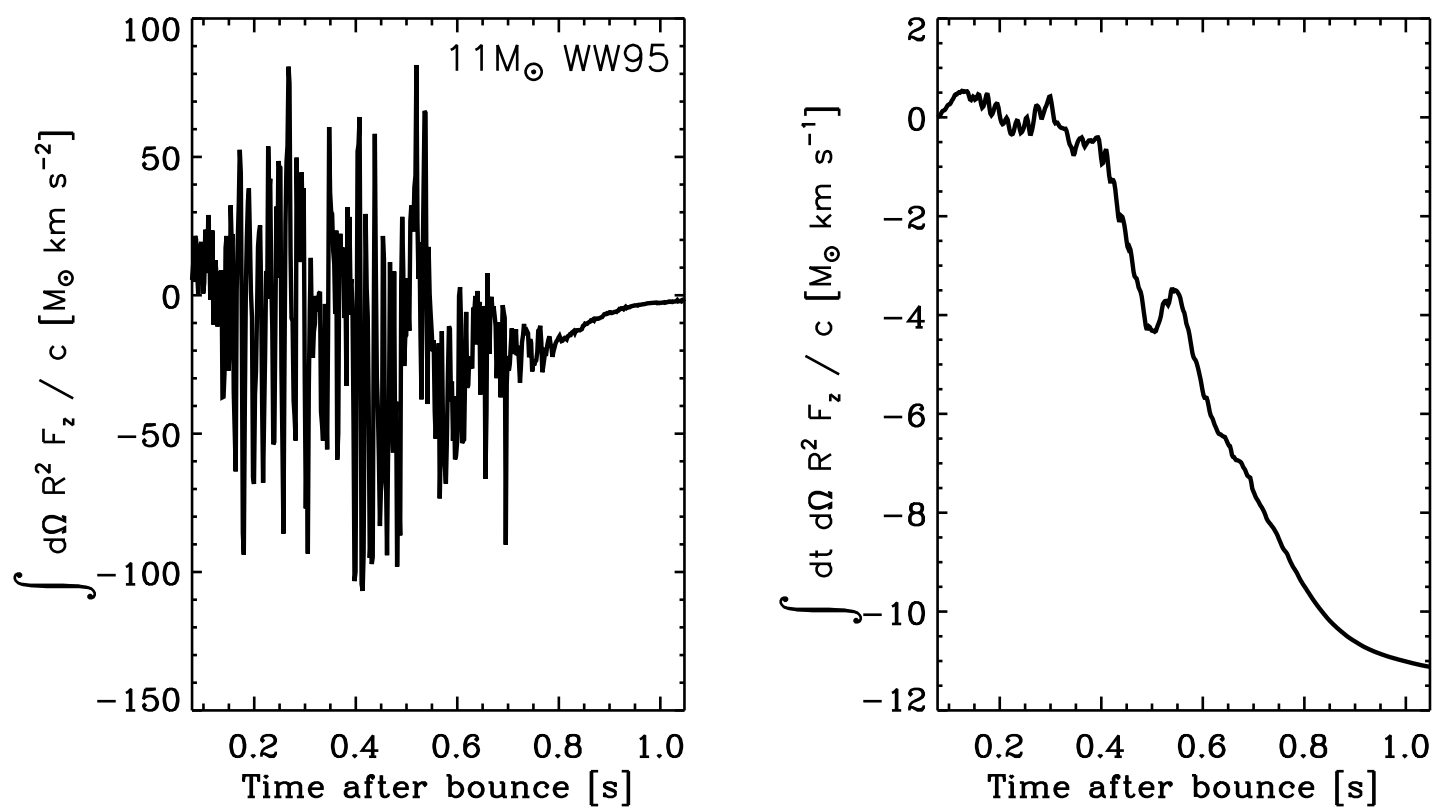

Figure 4: Left: Angle-averaged force due to the emergent neutrinos as a function of time after bounce for the 11-M $\odot$ model of WW95, from the calculations of Burrows et al.[1]. Right: Time integral (impulse) of the instantaneous force shown in the left panel as a function of time after bounce. Since by $\sim 1$ second after bounce a $1.4 \mathrm{M}_{\odot}$ neutron star had formed, the kick imparted as a result of the anisotropy of the neutrino emissions was no more than $\sim 10 \mathrm{~km} \mathrm{~s}^{-1}$ at that time, but was still increasing at the end of the calculation. 